\title{
Control of the intensity of a bathtub vortex by acting on the upstream flow
}

\author{
Azeddine Kourta $^{1, a}$, Yann Recoquillon ${ }^{1,2}$ And Emmanuelle Andrès $^{2}$ \\ 1 University Orléans, INSA-CVL, PRISME, EA 4229, 8 rue Léonard de Vinci, 45072 Orléans, France \\ 2 Renault, DREAM / DELT'A, 78288 Guyancourt, France
}

Received 8 December 2014, Accepted 19 June 2015

\begin{abstract}
In a motor vehicle, the cowl box is a volume located at the bottom of the windshield. It collects rainwater and drains it to provide clean dry air to the passenger compartment through the ventilation system. When rainwater accumulates in the box, a bathtub vortex forms above the drain pipe. This vortex sucks up air into the pipe and creates an air core that decreases the water drain rate in the pipe. This increases the water level in the box and can cause a water overflow into the ventilation system. The behavior of this bathtub vortex was experimentally studied using a simplified geometry representative of a real cowl box. The inlet water flow rate was controlled and a capacitive probe measured the water level in the box. The flow was studied using Particle Image Velocimetry to measure the velocity field around the vortex. The flow pattern is described using these data. Due to geometry and inlet conditions, the upstream flow forces the vortex counter-clockwise. It also shifts the vortex axis away from the drain axis. In this configuration, the upstream flow is strongly asymmetric and feeds the vortex using less than half the width of the box. Based on these observations, a device was tested in order to reduce the vortex intensity and consequently the water level. Resulting velocity fields showed a better distribution of the upstream flow. The vortex intensity decreased by up to $55 \%$ and the water level by up to $53 \%$. While there was still a vortex with an air core, the water level was therefore significantly reduced. These results are particularly interesting for cowl box design: as this device can guarantee a lower water level, the cowl box depth can be reduced and space can be saved.
\end{abstract}

Key words: Cowl box / bathtub vortex / PIV measurements / control water level

\section{Introduction}

This work focuses on the impact of the bathtub vortex phenomenon on the water flow inside the cowl box of a motor vehicle.

In a motor vehicle, a cowl box is a volume located at the bottom of the windshield (Fig. 1). It is designed to provide clean fresh air to the Heating, Ventilation and Air Conditioning unit (HVAC). It is also designed to avoid water ingestion into the HVAC unit, which can cause a variety of issues from passenger discomfort to the breakdown of the HVAC unit. To achieve this goal under rain conditions, the cowl box has to collect the rainwater coming from the windshield and drain it outside the car. The ventilation of the passenger cell is managed by the HVAC unit which sucks up air in the cowl box. An airflow is created and fresh air enters the cowl box through the top cowl grid (Fig. 1). The box is designed as a tranquilization

\section{Nomenclature}

\begin{tabular}{|ll|}
\hline$D$ & Drain hole diameter \\
$F r$ & Froude number \\
$g$ & Gravitational acceleration \\
$h$ & Water level \\
$H$ & Average water level \\
$L_{\mathrm{c}}$ & Characteristic length \\
$l$ & Box width \\
$Q_{\mathrm{wi}}$ & Water inlet flow rate \\
$R e$ & Reynolds number \\
$R$ & Radius \\
$T$ & Time \\
$U_{\mathrm{c}}$ & Characteristic velocity \\
$u$ & Radial velocity component \\
$v$ & Tangential velocity component \\
$\vec{V}$ & Velocity vector \\
\hline
\end{tabular}

\footnotetext{
a Corresponding author:
}

azeddine.kourta@univ-orleans.fr 


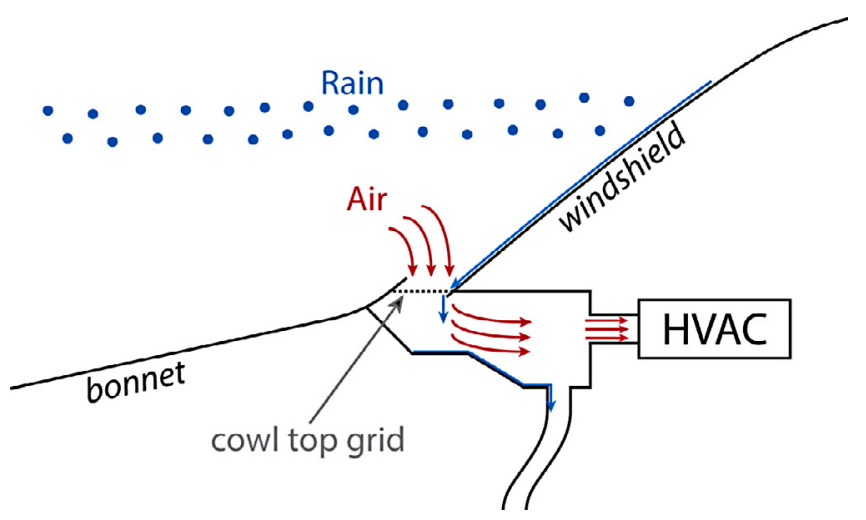

Fig. 1. Sketch of cowl box.

(HVAC: Heating, Ventilation and Air Conditioning)

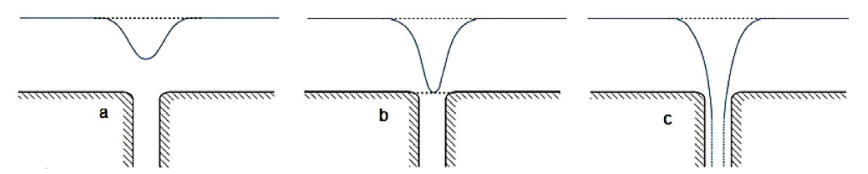

Fig. 2. Different regimes: (a) subcritical, (b) critical and (c) supercritical regime.

chamber: the airflow coming from the top grid is slowed down and the water contained in the airflow is collected by gravity. The volume of water collected at the bottom of the cowl box is drained outside the car by pipes in secured areas to protect electronics or other water-sensitive parts in the engine cell. The pipes are designed to guarantee a drain rate greater than the rainwater inlet flow rate to avoid an increase in the water level in the box. Due to strong design constraints on other elements around the cowl box, it is frequently difficult to use larger drain pipes.

As in a draining bathtub, a vortex appears above the drain pipes in the cowl box. The presence of a bathtub vortex can strongly damage the cowl box performance.

Stepanyants and Yeoh [1] defined three different vortex flow regimes. Subcritical vortices correspond to small dents on the free surface (Fig. 2a), less than the water height. When the vortex tip reaches the drain hole, the flow is in critical regime (Fig. 2b). The supercritical regime occurs when a strong vortex creates an air core extending through the drain pipe (Fig. 2c) (see also Recoquillon [2]). In the cowl box, the vortex is in supercritical regime (Fig. 3). Part of the pipe section is occupied by the air core, reducing the effective section used to drain water. This flow characteristic reduces the water drain rate and increases the water level in the box. This can lead, in the worst case, to overflow into the ventilation system.

The bathtub vortex is a common phenomenon in daily life, but it is still not totally understood. Andersen et al. $[3,4]$ described the specific flow structure inside the vortex. This structure was also observed by Echavez and McCann [5]. These studies, like most of the work on the bathtub vortex, focused on the subcritical and critical regimes. Only a few contributions, such as the ones by Stepanyants and Yeoh [1] or Forbes and Hocking [6], dealt

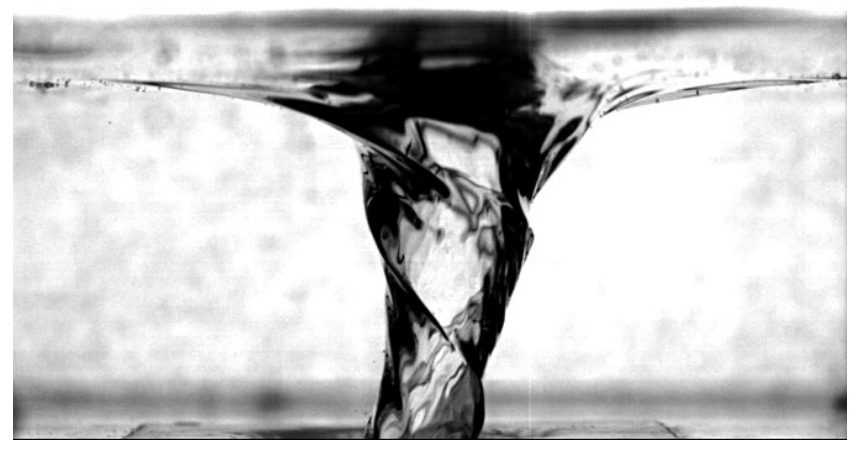

Fig. 3. Vortex above a drain pipe.

with the specific case of the supercritical vortex. In the cowl box, this supercritical regime decreases the draining capabilities of the box and therefore needs to be investigated.

A bathtub vortex has a negative effect on the capacity of the cowl box to exit the flow and hence increases the level of the flow inside the geometry. In order to limit the negative effects of the bathtub vortex, it is important to modify the internal geometry of the cowl box. One device that can improve the exhaust capacity of the cowl box is an adapted grid placed upstream of the exhaust. In the present study, two different grids were tested and interesting results were obtained.

\section{Experimental set-up}

An actual cowl box has a complex geometry and is awkward to access. To be able to study the bathtub vortex, a simplified cowl box was designed.

\subsection{Simplified cowl box geometry}

For better optical access, the cowl box is represented as a Plexiglas parallelepiped $1.24 \mathrm{~m}$ long, $0.24 \mathrm{~m}$ wide and $0.24 \mathrm{~m}$ high, corresponding to a full scale box (Figs. 4 and 5). A plane corresponding to the windshield is placed on the top side with an angle of $35^{\circ}$. At the bottom of this plane, a cowl top grid collects water flowing down from the plane and lets it run into the box. On the other side of the box, the drain pipe is located at equidistance of each side wall. It has a length of $0.1 \mathrm{~m}$ and its inner diameter is $D=26 \mathrm{~mm}$. The edge between the bottom of the box and the pipe has a rounded shape with a radius of $10 \mathrm{~mm}$.

The cowl box is placed above a buffer tank that stores water and collects the flow coming from the box. A pump takes water from the tank and provides it to the box through a device creating a water layer on the windshield. A flowmeter placed on the pipe between the pump and the windshield measures the water inlet flow rate $Q_{\text {wi }}$. The flowmeter is designed to measure a flow rate from 0.3 to $37.61 . \mathrm{min}^{-1}$ with an accuracy of $\pm 0.371 . \mathrm{min}^{-1}$. 


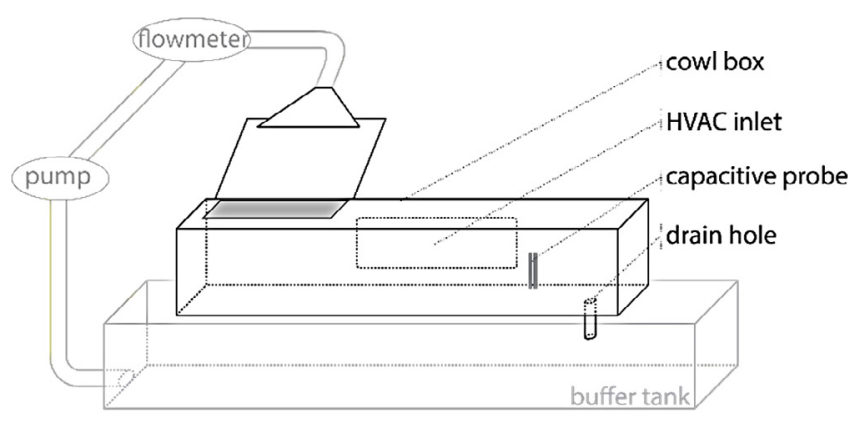

Fig. 4. Sketch of the experimental setup.

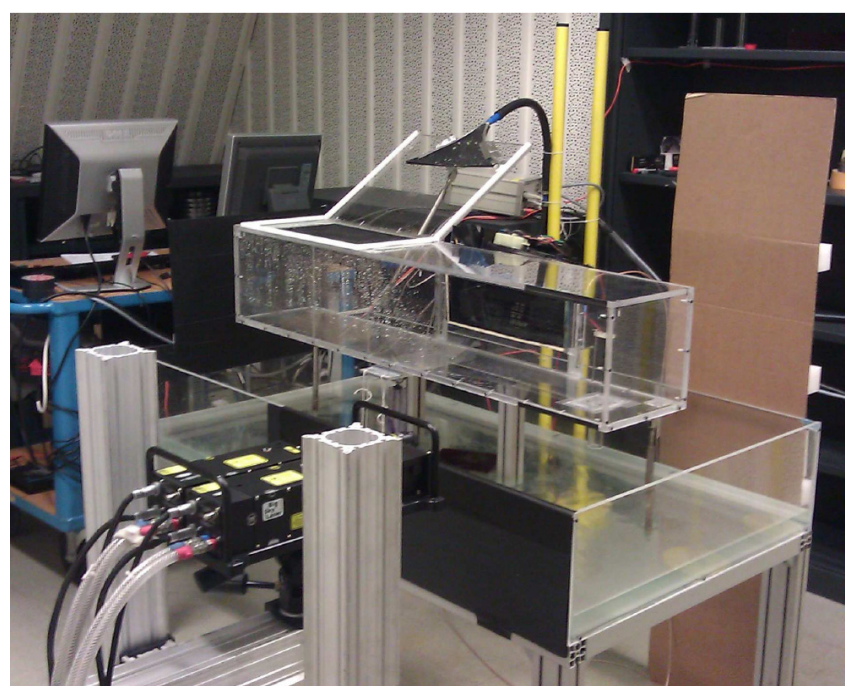

Fig. 5. Photo of the experimental setup.

To monitor the water level $\mathrm{h}$ in the cowl box, a capacitive probe is placed on a side wall. The probe can measure a water level up to $0.1 \mathrm{~m}$ with an accuracy of $\pm 2 \times 10^{-3} \mathrm{~m}$. Both flow rate $Q_{\text {wi }}$ and water level h are recorded at a frequency of $10 \mathrm{~Hz}$.

\subsection{Particle image velocimetry (PIV)}

In addition to monitoring the water level and inlet flow rate, Particle Image Velocimetry (PIV) was used to measure the velocity fields in the vortex area. The PIV device contained two Nd-Yag lasers producing two pulses delayed by $900 \mu$ s with a wavelength of $532 \mathrm{~nm}$. The camera used here had a resolution of $2048 \times 2048$ px. 200 pairs of images were recorded. Water flowing into the cowl box was seeded by nylon particles with a mean diameter of $4 \mu \mathrm{m}$. Acquisition and post-processing were conducted with Insight $3 \mathrm{G}$ software by TSI.

The configuration used here made it possible to measure the radial and tangential velocity components of the vortex flow. Lasers were placed in front of the cowl box, creating a horizontal laser sheet passing through the water layer above the drain pipe (Fig. 6). Images were recorded with a camera placed above the box. Due to the air/water interface in the vortex core, the laser sheet is diffused. Behind the air and the rear part of the vortex, a shadow

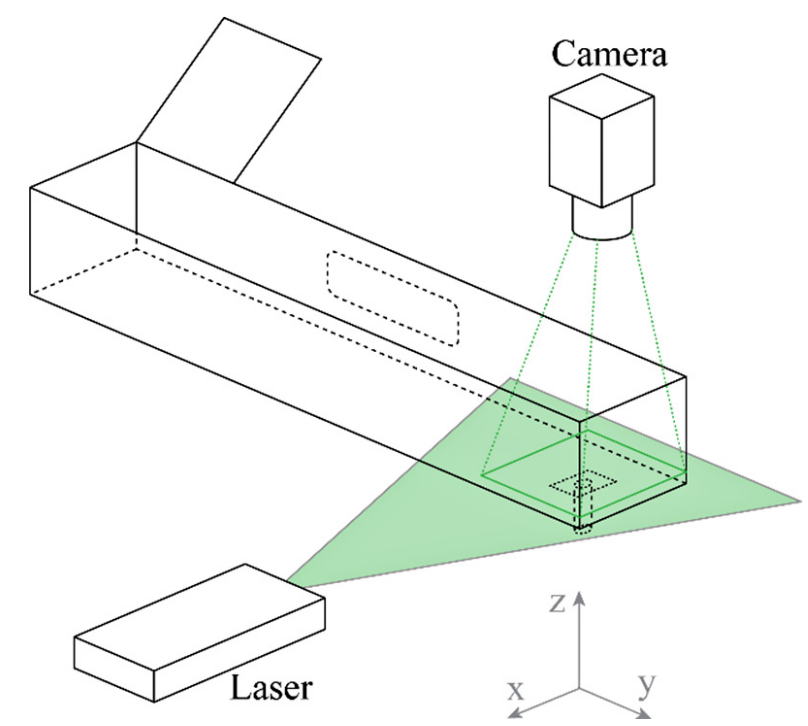

Fig. 6. Sketch of the PIV setup.

zone existed and there was not enough light to illuminate particles. In this zone, errors of measurements were important. For this reason, only the front half part of the velocity field was used for the analysis (upper part in the figures).

These measurements were carried out in stationary states. The testing procedure was as follows:

$-t=0 \mathrm{~s}$ : the box is empty. The pump is started and water starts to flow into the box. The water level increases. The water level and inlet water flow rate are recorded.

$-t \approx 200 \mathrm{~s}$ : the water level stabilizes.

$-t=400 \mathrm{~s}$ : the water level is stable. PIV acquisition is started.

Fluctuations in the size and position of the air core were observed during the measurements. The amplitude of these fluctuations was small compared to the size of the air core. For each configuration tested, the 200 instant velocity fields obtained by PIV were averaged. Only the average velocity fields will be analyzed in this paper. The average air core position can vary, depending on the flow configuration. To facilitate comparison between the results of different configurations, the vortex frame was defined as the reference frame: its origin is the vortex axis.

\section{Results}

In this paper, all the results presented were measured at $Q_{\text {wi }}=161 . \mathrm{min}^{-1}$. The laser sheet was placed at $50 \%$ of the average water level $H$.

\subsection{Reference flow pattern}

The bathtub vortex was previously studied experimentally [7]. The results obtained provided a description of the bathtub vortex, the three velocity component profiles, water height and the dimension of the air 
Table 1. Dimensionless parameters at $Q_{\mathrm{wi}}=16 \mathrm{l} \cdot \mathrm{min}^{-1}$.

\begin{tabular}{ccccc}
\hline Zone & $L_{\mathrm{c}}(\mathrm{mm})$ & $U_{\mathrm{c}}$ & $R e$ & $F r$ \\
\hline Box & $H=46.6$ & $Q_{\mathrm{wi}} /(H l)$ & 1100 & 0.035 \\
Drain pipe & $D=26$ & $4 Q_{\mathrm{wi}} /\left(\pi D^{2}\right)$ & 13000 & \\
\hline
\end{tabular}

(a)

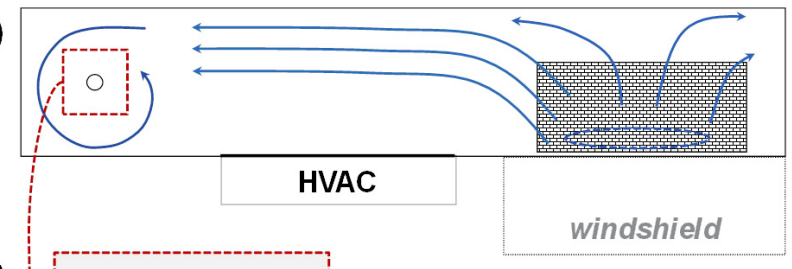

(b)

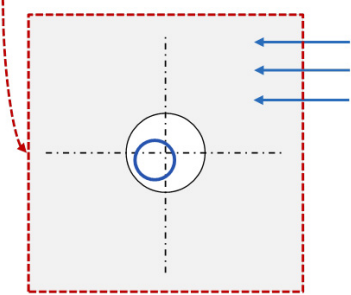

Fig. 7. Flow pattern: (a) global flow pattern (top view), (b) zoom on the drain hole area. The blue circle represents the position of the air core.

core inside the vortex. The flow can be characterized by two dimensionless parameters: Reynolds $(R e)$ and Froude $(F r)$ numbers. In fact, to respect kinematic and dynamic similarity, two dimensionless numbers appear in the governing momentum equations in dimensionless form: one in the viscous term and compares viscous to inertial forces (Reynolds number), another one in the gravity term and compares gravity to inertial forces (Froude number). Models with the same geometry but different sizes and with the same Reynolds and Froude numbers correspond to the same fluid dynamic solution. These two numbers are:

$$
R e=\frac{U_{C} L_{C}}{\nu} \quad F r=\frac{U_{C}}{\sqrt{g L_{c}}} .
$$

These numbers are based on characteristic velocities and lengths. Both numbers are computed in the box by using the average water level as the characteristic length. In addition, the Reynolds number can be computed in the drain pipe by using its diameter as the characteristic length. The corresponding characteristic velocities are reported in Table 1 . Both velocities are computed by using the water inlet flow rate divided by the corresponding surface. For the box this surface is equal to its length multiplied by the average water level. For the pipe it is based on the diameter.

The dimensionless parameters in Table 1 show a laminar flow in fluvial motion in the box. The flow becomes turbulent in the drain pipe. These parameters were used to compare the different configurations studied in this paper.

The flow pattern is plotted in Figure 7, showing a top view of the flow pattern and the drain hole area with the position of the air core of the bathtub vortex. (a)

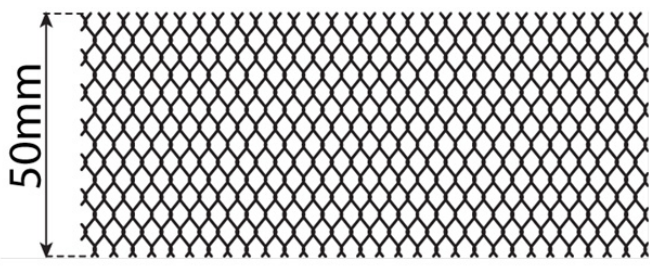

(b)

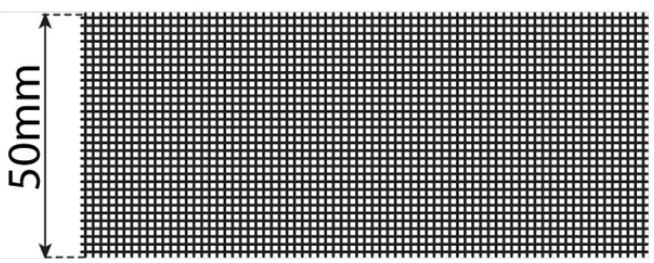

Fig. 8. Grids used in the cowl box: (a) Coarse grid $3 \mathrm{~mm}^{2}$, (b) Fine grid $0.5 \times 0.5 \mathrm{~mm}^{2}$.

The reference flow configuration has a pattern imposed by the water inlet condition: water flowing from the plane passes through the cowl top grid and falls into the box. It creates a flow toward the drain hole mostly concentrated along the opposite side wall. This pattern forces the vortex to flow counter-clockwise. In Figure 7b, the upstream flow comes from the top right of the sketch. It creates a shift in the vortex axis toward the bottom left of the drain pipe axis.

Some vortices are subject to strong position fluctuations. This effect, called "vortex meandering" [8], is a major difficulty for experimental measurements. Here the vortex shows only small amplitude fluctuations compared to the shift of its axis. The average shift is $1 \mathrm{~mm}$ on the $x$ axis and $3 \mathrm{~mm}$ on the $y$ axis. As the vortex frame is used as a reference, this shift has no effect on the data analysis.

These results show the characteristic pattern of the reference flow. The upstream flow is asymmetric and has a strong influence on the vortex flow since it imposes the position and direction of rotation of the vortex.

\subsection{Grid controlling the upstream flow}

To improve the capacity of the cowl box to evacuate the water and hence not exceed the safety limit, we decided to add a grid upstream of the exhaust hole. The function of the grid is to better homogenize the upstream flow and delay the air core of the bathtub vortex. Two different grids, a coarse and a fine one, were tested (Fig. 8). The coarse grid with cells of $3 \times 3 \mathrm{~mm}^{2}$ was in a metal and the fine grid with cells of $0.5 \times 0.5 \mathrm{~mm}^{2}$ was in scourer (sponge) and was $6 \mathrm{~mm}$ thick. Both grids have the same length equal to the width of the box and a height of $50 \mathrm{~mm}$. The height of the grid had to be at the least $50 \mathrm{~mm}$. In this case, its upper side was higher than the interface between water and air in all cases and hence did not affect the water.

By using a coarse grid, a $1 \mathrm{~mm}$ difference in the water level between upstream and downstream was observed. This difference was $10 \mathrm{~mm}$ with the fine grid. 


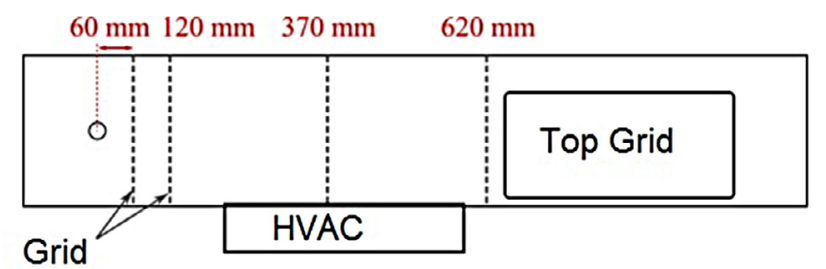

Fig. 9. Parametric study of the grid position.

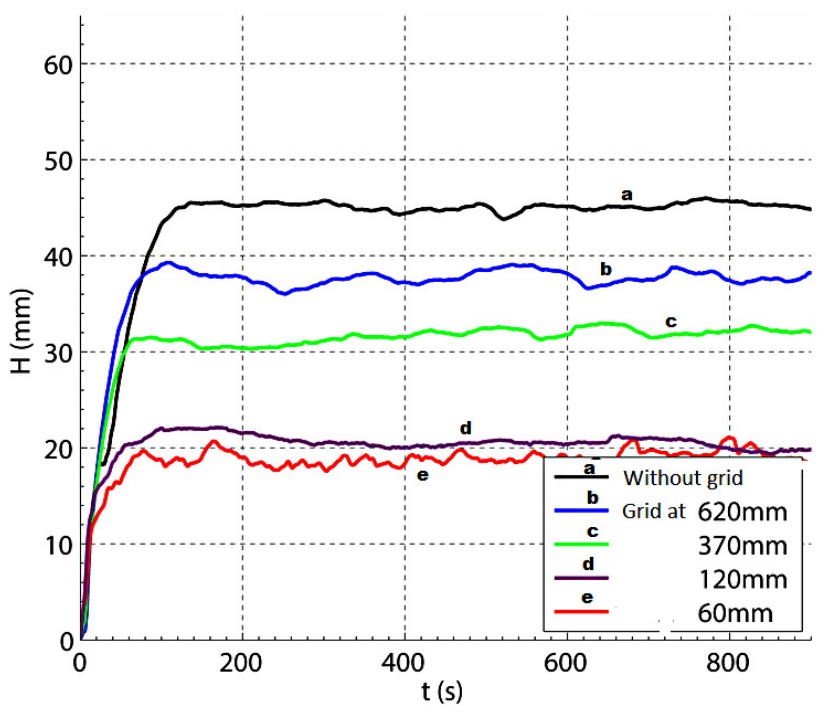

Fig. 10. Water level in the cowl box for different grid positions.

Considering the velocity as having a uniform distribution (computed by using the flow rate) and using the Bernoulli equation, the pressure loss obtained was $\Delta P=9.8 \mathrm{~Pa}$ for the coarse grid and $\Delta P=97 \mathrm{~Pa}$ for the fine one.

\subsection{Parametric study of the grid position}

To determine the optimal position upstream of the pipe, four different positions of the grid were tested (Fig. 9). Figure 10 gives the water level obtained for the four cases. The water level decreased with the upstream position up to $120 \mathrm{~mm}$. Beyond this position the level did not change. The most efficient position, therefore, was when the grid was placed $120 \mathrm{~mm}$ from the drain pipe. This position of the grid corresponds to the case for which the drain pipe is at the center of the square cavity delimited by the three box walls and the added grid. The reference length to be used for similarity criteria seems to be the width of the cowl box $(l=240 \mathrm{~mm}$ in the present case). $l$ is also twice the distance between the drain pipe axis and the wall box behind the hole.

To study the performance of the coarse and fine grids, the grid was positioned upstream of the hole, $120 \mathrm{~mm}$ from its axis (Fig. 11).

Figure 12 shows the water level in the case without the grid and in the two cases with each grid. In each case three measurements were made. For each configuration,

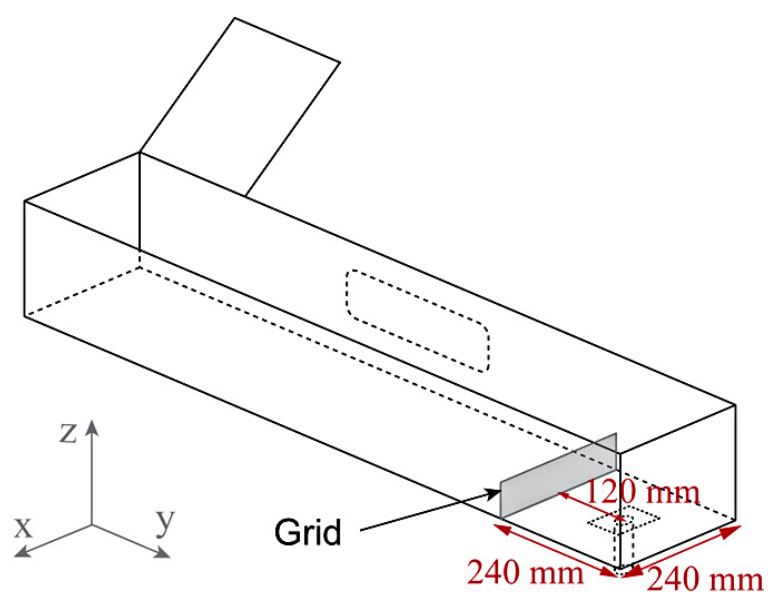

Fig. 11. Grid position used to compare the two grids.

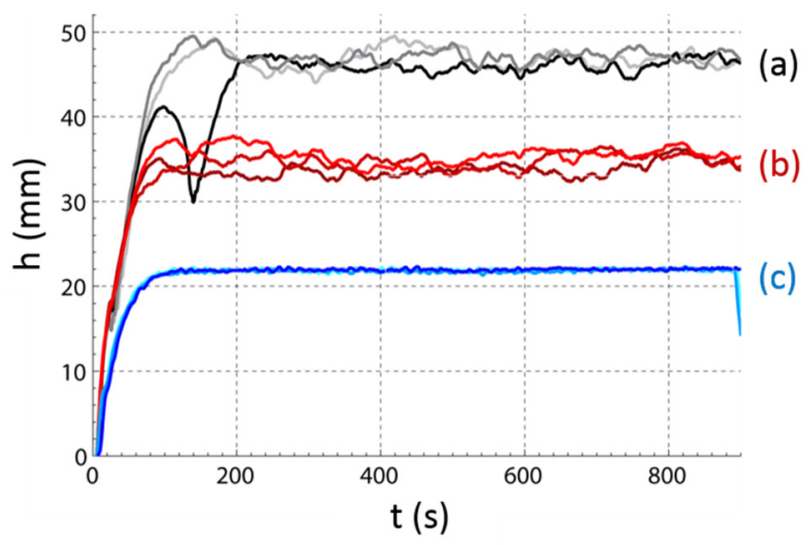

Fig. 12. Water level versus time : (a) without grid, (b) with coarse grid, (c) with fine grid.

Table 2. Froude number with and without grid at $Q_{\mathrm{wi}}=$ $161 . \mathrm{min}^{-1}$.

\begin{tabular}{ccc}
\hline Case & $H(\mathrm{~mm})$ & $F r$ \\
\hline Reference & 46.6 & 0.035 \\
Coarse grid & 34.6 & 0.055 \\
Fine grid & 21.9 & 0.109 \\
\hline
\end{tabular}

the water level was computed by averaging the three measurements in the range $200<t<880 \mathrm{~s}$. The values obtained were $46.6 \mathrm{~mm}$ in the reference case, $34.6 \mathrm{~mm}$ with the coarse grid and $21.9 \mathrm{~mm}$ with the fine grid. They correspond to a decrease in the water level of $25.7 \%$ for the former and $53 \%$ for the latter. The use of the grid had a beneficial effect as it decreased the water level in the cowl box.

The results presented here were all obtained for the same inlet water flow rate $Q_{\mathrm{wi}}=16 \mathrm{l} \cdot \mathrm{min}^{-1}$. As flows were in stationary state, the drain rate equaled the inlet water flow rate, leading to the same Reynolds numbers for the 3 cases. Due to the decrease in the water level, the Froude number in the box increased with the grid. However, the results in Table 2 show that the flow remained in fluvial motion for the 3 cases. 


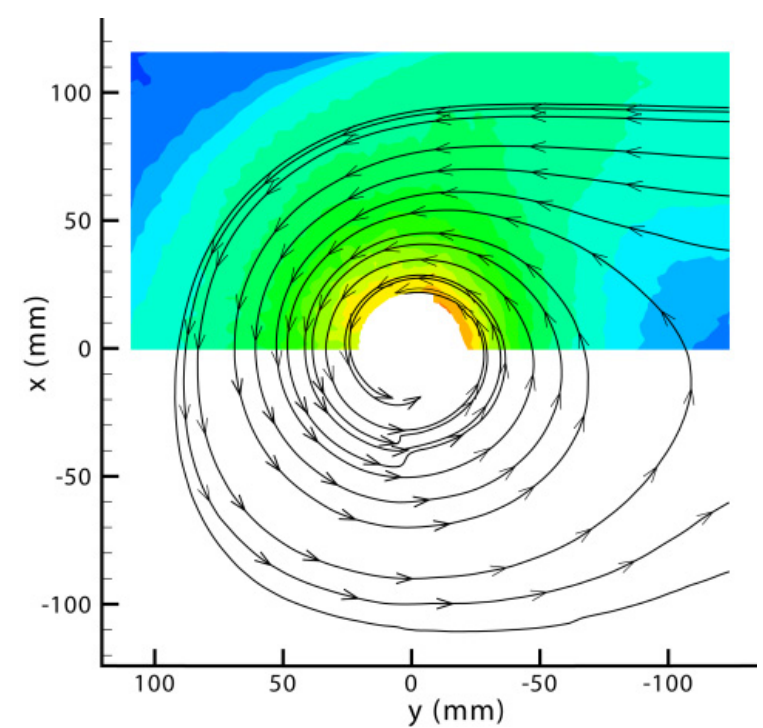

(a)

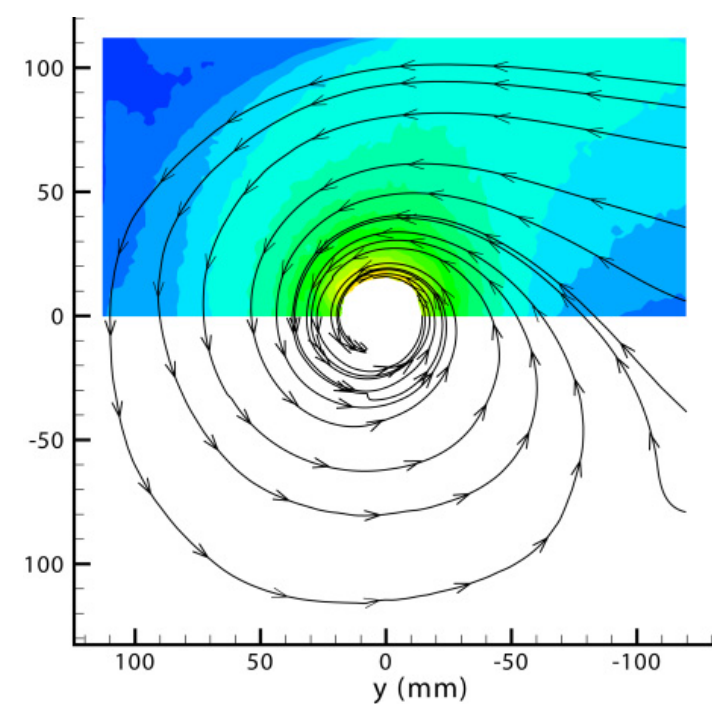

(b)

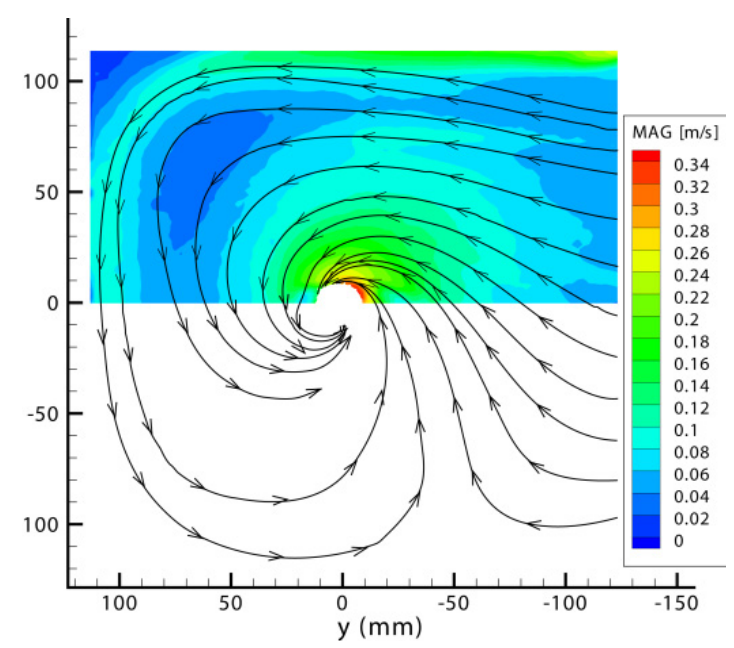

(c)

Fig. 13. Velocity magnitude and stream lines: (a) without grid, (b) coarse grid, (c) fine grid.
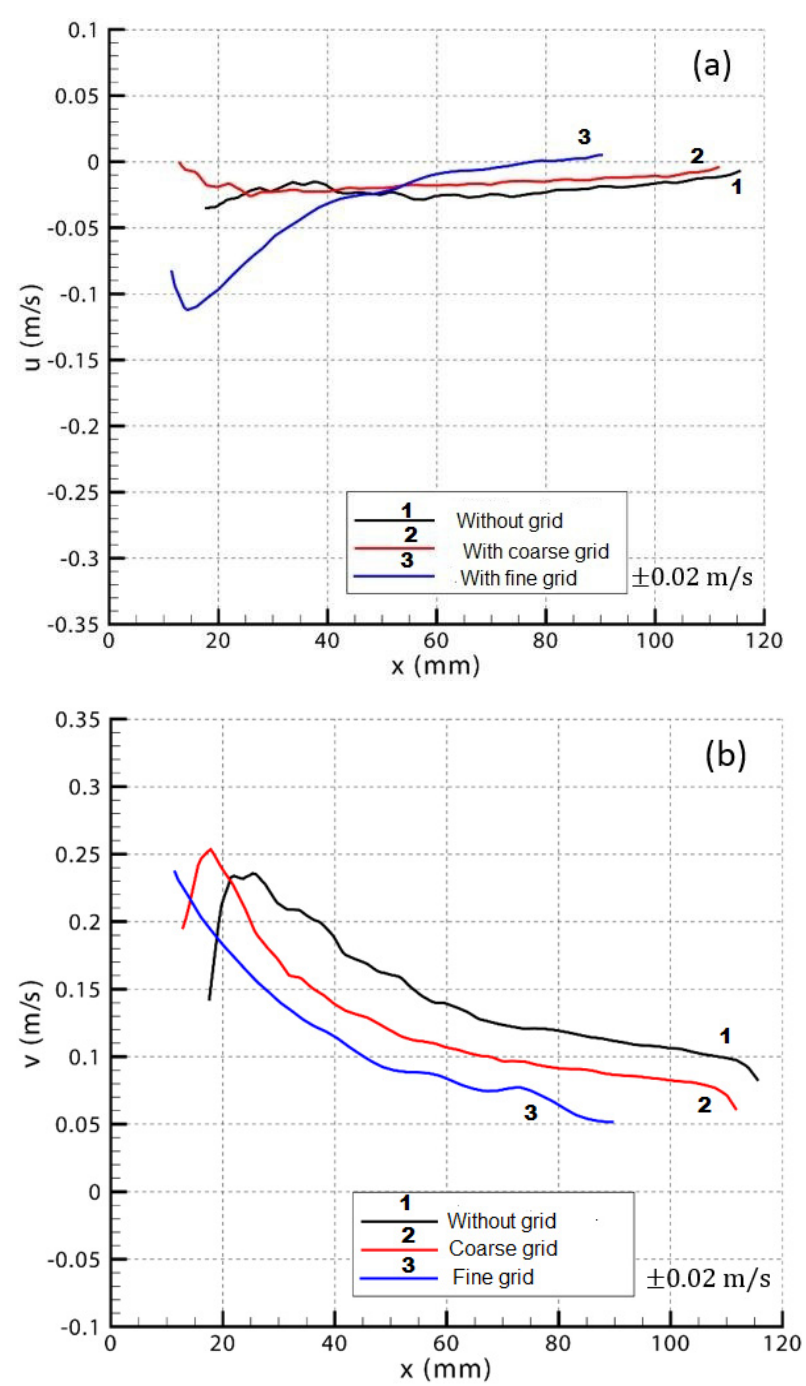

Fig. 14. Velocity profiles: (a) radial component $u$, (b) tangential component $v$.

The use of the grid significantly decreased the water level. In order to characterize the grid effect, it is important to analyze the flow. For the same water level, the flow pattern was analyzed by using PIV. These measurements provide flow visualizations and velocity profiles. In Figure 13 the iso-surface of velocity magnitude and streamlines for the three cases are plotted. In case (a), without the grid, there were as many upstream as downstream streamlines (though in opposite directions). With the coarse grid, the upstream flow tended to become parallel and to flow over the drain hole. On the bottom part of the box with respect to the hole, the reverse flow was blocked by the upstream flow. This distribution led to a concentration of the velocities around the vortex, whereas on the periphery of the vortex, the velocities were weaker.

With a fine grid that induces a greater pressure loss, the upstream flow was more uniform and was distributed across the whole width of the box. The flow was strongly opposed to the reverse flow, thus concentrating the maximum velocities around the air core. 


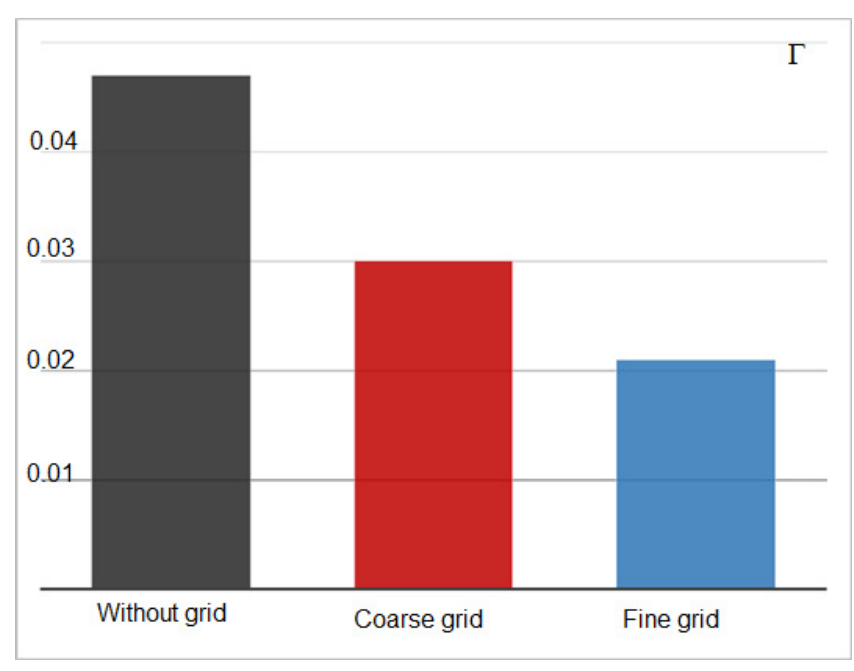

Fig. 15. Circulation $\Gamma$ with and without grid $\left(\mathrm{m}^{2} \cdot \mathrm{s}^{-1}\right)$.

Figure 14 shows the velocity component profiles. For the radial component, it can be seen that with the fine grid, little change was obtained. With the coarse grid a substantial change can be observed. The maximum increased with the grid and moved toward the axis. For the tangential component, the grid effect can be clearly observed. With the grid the tangential velocity component decreased and then moved toward the vortex axis.

To evaluate the intensity of the vortex, the circulation $\Gamma$ can be computed by integrating the velocity over a closed contour $C$ :

$$
\Gamma=\oint_{C} \vec{V} \cdot \mathrm{d} \vec{l}
$$

$\vec{V}$ is the velocity vector.

As PIV provides two-dimensional velocity fields, it is possible to compute $\Gamma$ over a circle of radius $r$ concentric with the vortex:

$$
\Gamma=\int_{-r}^{r} u \cdot \mathrm{d} l_{x}+\int_{-r}^{r} v \cdot \mathrm{d} l_{y}
$$

Circulation was computed for a range of circles with $12 \mathrm{~mm} \leq r \leq 120 \mathrm{~mm}$. The circulation increased with $\mathrm{r}$ and tended to a constant value for $60 \mathrm{~mm} \leq r \leq 100 \mathrm{~mm}$. For $r>100 \mathrm{~mm}$, it decreased due to the boundary effect. The maximum value of the circulation, reached in the range $60 \mathrm{~mm} \leq r \leq 100 \mathrm{~mm}$, is presented in Figure 15 for the three cases. The circulation decreased with the grid and this decrease was greater with the fine grid than with the coarse one.

\section{Conclusions}

In this work the cowl box water level and its connection with the bathtub vortex were studied. To optimize the cowl box water exhaust, a control was applied to decrease the air core of the vortex by adding a grid inside the box. Two grids were tested. Experimental measurements show that both grids significantly improved the cowl box water exhaust. The optimal grid position corresponded to $120 \mathrm{~mm}$ from the drain pipe axis. With the fine grid this improvement was better than with the coarse grid. In this case, the water level decreased by up 53\%. At the same time the intensity of the bathtub vortex and the diameter of the air in its core decreased strongly (by more than 50\%). For this control system, a patent application (FR2985689) has been filed. The solution to use grid inside the cowl box is retained by Renault to be probably used in the future cars.

\section{References}

[1] Y. Stepanyants, G. Yeoh, Stationary Bathtub Vortices and a Critical Regime of Liquid Discharge, J. Fluid Mech. 604 (2008) 77

[2] Y. Recoquillon, Étude expérimentale et numérique des écoulements diphasiques dans la boite à eau d'un véhicule automobile, Ph.D. Thesis, University of Orléans, France

[3] A. Andersen, T. Bohr, B. Stenum, J. Juul Rasmussen, B. Lautrup, Anatomy of a bathtub vortex, Phys. Rev. Lett. 91 (2003) 104502

[4] A. Andersen, T. Bohr, B. Stenum, J. Juul Rasmussen, B. Lautrup, The bathtub vortex in a rotating container, J. Fluid Mech. 556 (2006) 121-146

[5] G. Echávez, E. McCann, An experimental study on the free surface vertical vortex, Experiments in Fluids 33 (2002) 414-421

[6] L.K. Forbes, G.C. Hocking, The bath-plug vortex, J. Fluid Mech. 284 (1995) 43-62

[7] Y. Recoquillon, E. Andrès, A. Kourta, Experimental analysis of a bathtub vortex in a simplified cowl box of automotive vehicles, In ASME FEDSM 2012, Rio Grande, Puerto Rico, USA, July 2012. Paper No. FEDSM201272102, 2012, pp. 71-78

[8] L. Jacquin, D. Fabre, D. Sipp, E. Coustols, Unsteadiness, instability and turbulence in trailing vortices. C.R. Physique 6 (2005) 399-414 